\title{
Parsing Spontaneous and Evoked Neurotransmission on Both Sides of the Synapse
}

\author{
Patrick E. Rothwell \\ Graduate Program in Neuroscience, University of Minnesota, Minneapolis, Minnesota 55455 \\ Review of Chung et al.
}

At least three distinct forms of synaptic transmission have been described at excitatory and inhibitory synapses in the CNS. Under resting conditions, presynaptic terminals spontaneously release individual synaptic vesicles, producing miniature postsynaptic currents. When a presynaptic terminal is invaded by an action potential, two phases of evoked vesicle release ensue: a synchronous phase that is rapid and coordinated, and an asynchronous phase that is delayed and prolonged. Classic theories of quantal synaptic transmission assume that the same presynaptic vesicles are recruited by spontaneous and evoked neurotransmission, but emerging evidence suggests spontaneous and evoked release actually draw from two distinct pools of vesicles (Sara et al., 2005; Mathew et al., 2008; Fredj and Burrone, 2009), though this notion remains controversial (Groemer and Klingauf, 2007).

A recent report by Chung et al. (2010) in The Journal of Neuroscience has further clarified this issue by comparing the mechanism of synaptic vesicle recycling following evoked and spontaneous release. Recycling involves the retrieval of fused vesicles from

Received March 7, 2010; revised March 29, 2010; accepted April 6, 2010.

This work was supported by National Institutes of Health Grant DA23750 and the University of Minnesota Graduate School. I thank Dr. Mark J. Thomas for comments on this manuscript.

Correspondence should be addressed to Patrick E. Rothwell at his present address: Department of Molecular and Cellular Physiology and Nancy Pritzker Laboratory, Department of Psychiatry and Behavioral Science, Stanford University School of Medicine, 1050 Arastradero Road, Palo Alto, CA 94304. E-mail: prothwel@stanford.edu.

DOI:10.1523/JNEUROSCI.1177-10.2010

Copyright $\odot 2010$ the authors $\quad 0270-6474 / 10 / 306480-02 \$ 15.00 / 0$ the plasma membrane through endocytosis, which typically requires dynamin GTPase to split vesicle membrane from plasma membrane. The authors first confirmed that the GTPase activity of dynamin was reduced by dynasore, a selective inhibitor of dynamin 1 and 2. They also confirmed that dynasore impaired synaptic vesicle recycling in cultured hippocampal neurons, by infecting these neurons with synaptophysin-pHluorin. This construct is delivered to synaptic vesicles and fluoresces when vesicles fuse with the plasma membrane, but its fluorescence is normally quenched after vesicle endocytosis. Following brief stimulation, the synaptophysin-pHluorin signal persisted in the presence of dynasore, confirming that dynamin function is necessary for the normal retrieval of vesicles that fuse during evoked neurotransmission.

Chung et al. (2010) went on to examine whether dynasore impairs vesicle recycling in cultured hippocampal neurons, using whole-cell voltage-clamp recordings from individual pyramidal neurons. In contrast to this group's previous work on excitatory synapses (Sara et al., 2005), these experiments focused on inhibitory synapses, which prominently exhibit spontaneous vesicle release as well as synchronous and asynchronous evoked release. Evoked inhibitory currents were progressively reduced in the presence of dynasore, indicating that dynamin-dependent vesicle recycling is necessary to maintain evoked inhibitory neurotransmission. Surprisingly, exposure to dynasore for up to $1 \mathrm{~h}$ did not impair spontaneous inhibitory neurotrans- mission, even when the readily releasable vesicle pool was depleted by strong stimulation protocols. In fact, dynasore application significantly increased the frequency of miniature IPSCs (mIPSCs), and also tended to increase mIPSC amplitude following prolonged (i.e., $1 \mathrm{~h}$ ) application. The authors suggest that the increased mIPSC amplitude is consistent with a postsynaptic effect of dynasore, as changes in MIPSC amplitude are classically associated with changes in the number or sensitivity of postsynaptic receptors. However, this raises the possibility that dynasore may impair evoked transmission though a postsynaptic (rather than presynaptic) mechanism, since dynamin inhibition can interfere with the postsynaptic trafficking of neurotransmitter receptors (Carroll et al., 1999).

Chung et al. (2010) address this possibility by showing that dynasore treatment decreased the number of presynaptic vesicles detected in electron micrographs following stimulation, thus confirming that dynasore acts presynaptically to disrupt vesicle recycling. Furthermore, vesicles loaded with styryl dye during evoked transmission released dye more rapidly upon stimulation with high potassium than vesicles loaded during spontaneous transmission, suggesting that they belong to distinct populations. One caveat to interpreting these results is that styryl dye uptake does not distinguish between excitatory and inhibitory synapses. This issue was addressed in a separate experiment using hippocampal slice cultures from spH64 transgenic mice, which selectively express synaptophysin-pHluorin at 
inhibitory synapses. Dynasore had no significant effect on the fluorescent signal during spontaneous transmission, confirming that dynamin inhibition does not impair the recycling of spontaneously released vesicles at inhibitory synapses. These imaging data complement the cellular electrophysiology results in which inhibitory synaptic currents were pharmacologically isolated, thus confirming a specific effect at inhibitory synapses (cf. Mathew et al., 2008).

The styryl dye imaging experiments also addressed a discrepancy between a previous report from this group suggesting that spontaneous and evoked release originate from distinct vesicle pools (Sara et al., 2005), and another report suggesting that spontaneous and evoked release originate from the same population of vesicles (Groemer and Klingauf, 2007). Chung et al. (2010) first used the styryl dye FM2-10 to replicate their previous results (Sara et al., 2005), and also reproduced the results of Groemer and Klingauf (2007) using the styryl dye FM143. They showed that these contradictory results were related to high levels of neuronal activity in mature hippocampal cultures, which promote forms of bulk endocytosis that selectively capture FM1-43 (Clayton and Cousin, 2009). An additional experiment confirmed that spontaneous and evoked neurotransmission draw from distinct vesicle pools, by using FM2-10 and another spectrally separable styryl dye (FM5-95) to simultaneously image both spontaneous and evoked vesicle recycling and destaining.

One potential explanation for the dissociation between spontaneous and evoked vesicle pools is that these forms of release occur at distinct populations of synapses. However, the imaging studies conducted by Chung et al. (2010) demonstrate that spontaneous and evoked release occur together at the majority of synapses in cultured hippocampal neurons. Furthermore, previous work from this laboratory (Sara et al., 2005) suggests excitatory synapses also have separate vesicle pools for spontaneous and evoked release. This is intriguing because another recent report demonstrates distinct populations of postsynaptic NMDA receptors are activated by spontaneous and evoked glutamate release (Atasoy et al., 2008). In total, these results demonstrate a partition between spontaneous and evoked neurotransmission that spans both sides of excitatory synapses. It remains to be determined whether distinct postsynaptic populations of GABA receptors are activated by spontaneous and evoked vesicle release at inhibitory synapses.
These results (Atasoy et al., 2008; Chung et al., 2010) have important implications for studying the modulation of synaptic transmission and its modification by experience. For example, addictive drug exposure can modify excitatory and inhibitory synaptic function in several brain structures, and this may play a role in the development of drug addiction (Kauer and Malenka, 2007). Drug-induced adaptations in synaptic function are often studied using parameters of both evoked and spontaneous neurotransmission. The probability of neurotransmitter release from the presynaptic terminal is typically assessed using the paired-pulse ratio (PPR) of evoked responses, as well as the frequency of spontaneous miniature postsynaptic currents ("minis"). There are numerous examples where addictive drug exposure changes both parameters in parallel (e.g., Bonci and Williams, 1997), suggesting that the mechanisms underlying spontaneous and evoked release can be coordinately regulated. However, situations in which PPR and mini frequency do not change in parallel (e.g., Kourrich et al., 2007) may involve selective modification of either evoked or spontaneous release mechanisms. In these situations, mini frequency may be more directly related to the number of synapses, rather than the probability of evoked neurotransmitter release (Kourrich et al., 2007; Atasoy et al., 2008).

On the postsynaptic side, changes in mini amplitude are frequently used to corroborate postsynaptic changes in the sensitivity to evoked neurotransmitter release. However, discrepancies between mini amplitude and measures of evoked synaptic transmission may be related to distinct postsynaptic receptor populations activated by spontaneous and evoked neurotransmitter release (Atasoy et al., 2008). One way to circumvent this issue may be to measure evoked quantal events in the presence of strontium, which promotes asynchronous neurotransmitter release. Chung et al. (2010) demonstrated that synchronous and asynchronous release draw from the same presynaptic vesicle pool at inhibitory synapses, while Atasoy et al. (2008) showed that synchronous and asynchronous glutamate release activate the same postsynaptic population of NMDA receptors. Measurement of quantal events in the presence of strontium has the added advantage of restricting release to a defined population of stimulated synapses (Oliet et al., 1996). However, synchronous and asynchronous release are not similar in all respects, as they have different calcium requirements and may use distinct calcium sensors (Sun et al., 2007). Thus quantal events measured in strontium may access the same population of postsynaptic receptors activated by synchronous release, but through a presynaptic mechanism that differs from synchronous release.

The results of Chung et al. (2010) add to a picture of synaptic transmission that grows increasingly complex, with spontaneous and evoked neurotransmission drawing from distinct presynaptic vesicle pools with different recycling mechanisms, and engaging separate populations of postsynaptic receptors (Atasoy et al., 2008). This growing complexity presents a challenge for studying the modulation and modification of synaptic function, but also provides new opportunities for precisely defining the specific aspects of synaptic transmission that are altered in any given situation.

\section{References}

Atasoy D, Ertunc M, Moulder KL, Blackwell J, Chung C, Su J, Kavalali ET (2008) Spontaneous and evoked glutamate release activates two populations of NMDA receptors with limited overlap. J Neurosci 28:10151-10166.

Bonci A, Williams JT (1997) Increased probability of GABA release during withdrawal from morphine. J Neurosci 17:796-803.

Carroll RC, Beattie EC, Xia H, Lüscher C, Altschuler Y, Nicoll RA, Malenka RC, von Zastrow M (1999) Dynamin-dependent endocytosis of ionotropic glutamate receptors. Proc Natl Acad Sci U S A 96:14112-14117.

Chung C, Barylko B, Leitz J, Liu X, Kavalali ET (2010) Acute dynamin inhibition dissects synaptic vesicle recycling pathways that drive spontaneous and evoked neurotransmission. J Neurosci 30:1363-1376.

Clayton EL, Cousin MA (2009) The molecular physiology of activity-dependent bulk endocytosis of synaptic vesicles. J Neurochem 111:901914.

Fredj NB, Burrone J (2009) A resting pool of vesicles is responsible for spontaneous vesicle fusion at the synapse. Nat Neurosci 12:751-758.

Groemer TW, Klingauf J (2007) Synaptic vesicles recycling spontaneously and during activity belong to the same vesicle pool. Nat Neurosci 10:145-147.

Kauer JA, Malenka RC (2007) Synaptic plasticity and addiction. Nat Rev Neurosci 8:844-858.

Kourrich S, Rothwell PE, Klug JR, Thomas MJ (2007) Cocaine experience controls bidirectional synaptic plasticity in the nucleus accumbens. J Neurosci 27:7921-7928.

Mathew SS, Pozzo-Miller L, Hablitz JJ (2008) Kainate modulates presynaptic GABA release from two vesicle pools. J Neurosci 28:725-731.

Oliet SH, Malenka RC, Nicoll RA (1996) Bidirectional control of quantal size by synaptic activity in the hippocampus. Science 271:1294-1297.

Sara Y, Virmani T, Deák F, Liu X, Kavalali ET (2005) An isolated pool of vesicles recycles at rest and drives spontaneous neurotransmission. Neuron 45:563-573.

Sun J, Pang ZP, Qin D, Fahim AT, Adachi R, Südhof TC (2007) A dual-Ca2+-sensor model for neurotransmitter release in a central synapse. Nature 450:676-682. 